\title{
A influência do lúdico no processo de hospitalização infantil: a visão do palhaço
}

The influence of the play in infant hospitalization process: clown vision

\section{La influencia de la obra de teatro en el proceso de hospitalización infantil: la visión del payaso}

\author{
Gabriela Bovo SIMIONI 1 \\ Jussiara de ALMEIDA ${ }^{\mathbf{1}}$ \\ Larissa de Fátima SILVA ${ }^{1}$ \\ Tamara Aparecida de Souza LEITE ${ }^{1}$ \\ Sergio Fernando ZAVARIZE ${ }^{2}$ \\ Anderson MARTELLI ${ }^{3}$ \\ ${ }^{l}$ Graduanda em Psicologia pela Faculdade Municipal Professor Franco Montoro - Mogi Guaçu-SP, Brasil \\ ${ }^{2}$ Doutor em Psicologia pela Pontifícia Universidade Católica de Campinas, PUC-Campinas-SP, Brasil; \\ Professor da Faculdade Municipal Professor Franco Montoro-Mogi Guaçu-SP, Brasil \\ Diretor Acadêmico na Faculdade Mogiana do Estado de São Paulo, FMG - Mogi Guaçu - SP, Brasil \\ ${ }^{3}$ Professor da Faculdade Municipal Professor Franco Montoro - Mogi Guaçu-SP, Brasil; \\ Mestrando Ciências Biomédicas - UNIARARAS, Araras-SP, Brasil
}

\section{Resumo}

A internação hospitalar pode afetar o desenvolvimento da criança uma vez que esta se depara com um ambiente insalubre, causador de estresse e ansiedade, que a retira de seu cotidiano, apresentando uma situação de estranheza e abandono. Desta forma, considerando que tais fatores sejam mais relevantes no contexto infantil, este trabalho propôs a averiguação da visão humanizadora do palhaço, frente a sua atuação lúdica na tentativa de promover a integração de fatores essenciais para o bem-estar psíquico, físico e social da criança e do acompanhante no ambiente hospitalar. A metodologia utilizada foi uma pesquisa de natureza aplicada, sendo indutiva qualitativa, cujo objetivo foi coletar, por meio de questionário, relatos dos palhaços e médicos quanto às alterações emocionais e comportamentais ocorridas com os pacientes $\mathrm{e}$ acompanhantes durante as atuações e se são capazes de influenciar no tratamento desses pacientes. Participaram da pesquisa cinco indivíduos com idade entre 21 a 30 anos, de ambos os sexos que atuam como palhaços nos hospitais do município de Mogi Guaçu(SP)-Brasil e dois médicos que atuam na área pediátrica hospitalar. Nos resultados foi possível observar uma percepção positiva sobre os efeitos da atuação dos palhaços sobre a criança internada; concluindo que o ato de brincar pode ser extremamente benéfico na recuperação da criança e para a execução dos procedimentos clínicos nos leitos hospitalares.

Descritores: Hospitalização; Saúde da Criança; Ludoterapia; Jogos e Brinquedos; Adaptação Psicológica.

\begin{abstract}
Hospitalization can affect the child's growth once he or she is exposed to an unhealthy environment, causing of stress and anxiety, which takes him or her out of the normal routine, presenting a situation of strangeness and abandon. Therefore, considering such factors are even more relevant on childhood context this article proposed to investigate the humanized vision of the clown based on its ludic actuation attempting to promote the integration of essential factors to psycho, physical and social well-being of the children and its companion inside the hospital environment. The methodology used was a kind of applied research, and qualitative inductive, whose goal was to collect, through a questionnaire, reports of clowns and doctors as to emotional and behavioral changes with patients and caregivers during the performances and are able to influence in treating these patients. The participants considered were five individuals aged from 21 to 30 years, from both genders, which act as clowns at Mogi Guaçu(SP)-Brazil local hospital. As result, it has observed a very positive perception about the effects of clown actuation over the children; leading to the conclusion on the act of playing can be extremely benefic for child's healing and to the execution of clinical procedures on hospital facilities. Descriptors: Hospitalization; Child Health; Play Therapy; Play and Playthings; Adaptation, Psychological.
\end{abstract}

\section{Resumen}

La hospitalización puede afectar el desarrollo del niño, ya que se enfrenta a un ambiente insalubre, causando estrés y la ansiedad, que lleva desde su vida cotidiana, que presenta una situación extraña y abandono. Por lo tanto, teniendo en cuenta que estos factores son aún más relevantes en el contexto de los niños, este documento propone la investigación de la visión humanizada del payaso, fuera de su actuación lúdica en un intento de promover la integración de los factores esenciales para el bienestar mental, físico y social de los niños y compañera en el hospital. La metodología utilizada fue una especie de investigación aplicada, e inductivo cualitativa, cuyo objetivo era recoger, a través de un cuestionario, los informes de los payasos y los médicos como a los cambios emocionales y conductuales con pacientes y cuidadores durante las actuaciones y son capaces de influir en el tratamiento de estos pacientes. Los participantes fueron cinco individuos de 21-30 años, de ambos sexos, que actúan como payasos en los hospitales en la ciudad de Mogi Guaçu(SP)-Brasil y dos médicos que trabajan en el área de hospital pediátrico. En los resultados se observó una percepción positiva de los efectos de las acciones de los payasos en los niños hospitalizados; llegando a la conclusión de que el acto de jugar puede ser muy beneficioso para la recuperación del niño y para la realización de los procedimientos clínicos en camas de hospital.

Descriptores: Hospitalización; Salud del Niño; Ludoterapia; Juego e Implementos de Juego; Adaptación Psicológica.

\section{INTRODUÇÃO}

Percebe-se atualmente uma importante preocupação com as consequências do contexto ambiental sobre o desenvolvimento do ser humano como um todo. Dentre as várias situações que tem sido objeto de estudos, encontra-se o âmbito hospitalar, que é caracterizado como um ambiente insalubre e estressante. Este ambiente pode ser capaz de 
provocar medos e angustias em seus pacientes, o que afeta suas relações com o outro e sua capacidade de olhar para si próprio de maneira positiva ${ }^{1,2}$.

Particularmente, a criança torna-se vulnerável a esse ambiente e, cerca de cinco a dez por cento de todas apresentam em alguma época da infância, uma enfermidade prolongada ou incapacidade moderada ou grave ${ }^{3}$. Sendo assim, essas crianças ao serem hospitalizadas se encontram em condições de risco, tanto do ponto de vista orgânico, quanto nos aspectos emocional e educacional no curso do seu desenvolvimento. Para reduzir, ou neutralizar os efeitos adversos da hospitalização aos processos de desenvolvimento e aprendizagem da criança, algumas modalidades de assistência têm surgido com a finalidade de proporcionar experiências de atividades construtivas à criança hospitalizada ${ }^{2}$.

Diante deste contexto, a ludicidade surge como meio de promoção de estratégias adequadas para o enfrentamento da hospitalização, funcionando como um potencializador no processo de adaptação da criança frente às transformações que ocorrerão a partir do momento em que ela é submetida à internação ${ }^{4}$. Como forma de humanizar o ambiente hospitalar, vários meios são implantados, como brinquedotecas, contadores de histórias, palhaços e rodas de interação, o que demonstra o quão relevante se faz a permanência de instrumentos típicos da infância no hospital, para que não haja uma interrupção no processo de desenvolvimento da criança enquanto estiver hospitalizada ${ }^{5,6}$

Os médicos são considerados parte fundamental no processo de humanização, pois, possuem grande influência no ambiente hospitalar e interferem de forma direta $\mathrm{e}$ significativa no tratamento do paciente, desta forma torna-se essencial que estejam integrados e de acordo com estas novas formas de interações hospitalares.

\section{○ O lúdico e a hospitalização infantil}

A internação hospitalar afeta notavelmente o estado emocional da criança hospitalizada, levando-a ao medo e a altas taxas de estresse. Constitui uma situação que pode provocar uma grande sensação de abandono na criança. Esta tem que deixar sua casa, amigos e sua família, permanecendo em um ambiente estranho e quase sempre percebido como hostil. Muitas vezes seus pertences são retirados e, a ausência dos pais, devido a restrições médicas, é compreendida pela criança como abandono ${ }^{7}$.

Diversos transtornos são apontados diante da hospitalização, sendo eles: problemas de sono, perda de manifestações psicossomáticas, incapacidade de fazer contato e tomar iniciativas, agressão hostil, personalidade instável, desorientação, carência afetiva, distúrbios do comportamento e disfunções fisiológicas ${ }^{2,8}$. Diante disso, torna-se necessário que se desenvolvam estratégias para que se mantenham o desenvolvimento físico, psíquico e intelectual da criança, de forma normal ainda que esta esteja hospitalizada $^{2,7}$.

No Brasil, um avanço significativo no que diz respeito à criança hospitalizada é observado a partir da homologação do Estatuto da criança e do adolescente, a iniciar da Resolução $\mathrm{n}^{\circ} 41$ do CONANDA $^{9}$ - Conselho Nacional dos Direitos da Criança e do Adolescente, publicado em outubro de 1995, que definem nos seus artigos $8^{\circ}, 9^{\circ}$ e $10^{\circ}$ os direitos da criança e do adolescente hospitalizados. $\mathrm{O}$ artigo $8^{\circ}$ discorre sobre o direito da criança de ter conhecimento adequado de sua enfermidade, dos cuidados terapêuticos e diagnósticos, respeitando sua fase cognitiva, além de receber o amparo psicológico quando se fizer necessário. $\mathrm{O} 9^{\circ}$ pondera sobre o direito de desfrutar de alguma forma a recreação, programas de educação para a saúde, acompanhamento do curriculum escolar durante sua permanência no hospital. $\mathrm{O}$ artigo $10^{\circ}$ aborda os direitos de seus pais ou responsáveis participarem ativamente do seu diagnóstico, tratamento e prognóstico, recebendo informações sobre os procedimentos a que se submetem ${ }^{9}$.

O lúdico surge como método relevante, capaz de gerar maior eficácia em seu tratamento. Buscar alternativas apropriadas para reverter o estado ao qual a criança se encontra, ao passar por procedimentos rigorosos e que a leva ao medo e a retirada de sua rotina, torna-se essencial ${ }^{10}$. Na relação afetiva com o mundo, a ludicidade é um espaço de direito de toda criança, a fim de manter o exercício com as pessoas e objetos, tornando-se indispensável para a saúde física, emocional e intelectual da criança o que contribui para a eficácia e equilíbrio do adulto ${ }^{10}$.

A intervenção do palhaço no ambiente hospitalar é apontada como uma estratégia facilitadora no enfrentamento das doenças, promoção de um bem-estar físico e uma melhora da qualidade de vida da criança hospitalizada e dos familiares ${ }^{11,12}$. O humor permite à criança a exploração de fatos que por obstáculos pessoais não eram possíveis. Tal acesso permite a liberação de energia investida no problema, que então pode ser utilizada em outros pontos importantes da recuperação da saúde ${ }^{7}$. Sendo assim, o riso, desencadeado de forma frequente nas brincadeiras, induz a liberação de endorfina e diminui o estresse. Isto fortalece as respostas imunológicas e reduz as consequências negativas causadas pela exposição aos estímulos desagradáveis e dolorosos, que estão ligados ás intervenções médicas ${ }^{12}$.

Com a finalidade de zelar pelo bem estar da criança hospitalizada, em meio aos corredores e leitos infantis, têm surgido diversos grupos de atuação que levam a ludicidade ás crianças enfermas, as quais inserem a figura do palhaço como meio de alterar o aspecto negativo do ambiente hospitalar. Ao se deparar com esse profissional fazendo um implante de nariz vermelho, a criança pode ser capaz de transformar sua realidade em uma brincadeira. A seringa que para ela é sinônimo de dor, acaba se configurando como que um brinquedo ${ }^{13}$.

A participação do palhaço dentro do ambiente hospitalar exigiu que estes lapidassem suas técnicas de fazer sorrir, pois estas passaram a acontecer em um local mais regrado em que a brincadeira passaria a ser coisa séria, e teria que atender a todas as necessidades que a rotina hospitalar lhe impunha, necessitando de uma formação, preparo e, sobretudo escolher o trabalho com crianças no hospital, como uma forma de atuação profissional. O ator que escolhe o personagem de palhaço para atuar num hospital está definindo uma linguagem artística como instrumento de relação com o outro dirigindo ao que está saudável numa criança doente, no intuito de manter vivas as suas possibilidades de criar, sonhar e de rir. De fato, o mundo desse ator é bem diferente daquele do hospital, mas seu universo está muito próximo ao da criança. Essa proximidade cria rapidamente uma grande cumplicidade entre eles ${ }^{14}$.

Ao inserir no contexto do hospital, o palhaço, tem por objetivo realizar de forma profissional, brincadeiras e performances que são obtidas em treinamentos de suas habilidades de se relacionar com o outro e de se comunicar. Em complemento de suas atuações, utiliza técnicas de improviso a fim de promover o bem estar físico e mental, contribuindo para melhor qualidade de vida dos pacientes, 
acompanhantes e da equipe médica de forma geral. Esse tipo de intervenção pode, portanto, provocar a diminuição do estresse e da ansiedade gerada pelo ambiente ${ }^{12,15}$. Esta prática apresenta por finalidade ocasionar o rompimento da insalubridade hospitalar, promovendo assim, alívio e segurança aos que recebem a intervenção.

Levar a ludicidade aos corredores hospitalares tem sido considerado método de humanização, reinserindo o humano no funcionamento da instituição hospitalar; ou seja, prevenindo que o paciente seja diminuído a um ser que precisa de reparos em órgãos prejudicados ${ }^{2,16}$. O objetivo principal deste método é a consideração da dignidade do ser humano, tornando-se ainda mais relevante quando pensamos em pacientes infantis ${ }^{5,6}$

Segundo Carmo ${ }^{17}$, por este viés, alguns hospitais e profissionais da saúde, têm percebido que mais que tratar a doença se faz necessário tratar dos aspectos sociais e emocionais das pessoas internadas. Os males que afetam os pacientes, vão além das bactérias e vírus estão nos sentimentos da solidão, da tristeza e principalmente do medo. Dada à escassez científica em publicações, este trabalho apresenta como objetivo, averiguar a influência da figura do palhaço, sua atuação e as consequências geradas em pacientes infantis, avaliando alterações emocionais e comportamentais ocorridas com os pacientes e acompanhantes durante as atuações, verificando se as implicações são positivas ou negativas e se são capazes de influenciar no tratamento e procedimentos hospitalares.

\section{MATERIAL E MÉTODO}

\section{○ Amostragem}

O estudo envolveu 7 participantes, selecionados com base num método de amostragem por conveniência, segundo a disponibilidade dos profissionais para participar da pesquisa. $\mathrm{O}$ estudo contou, assim, com palhaços humanizadores $(n=5)$ escolhidos nos grupos de palhaços das duas ONG's existentes no municipio de Mogi Guaçu-SP UAI (Unidade de Alegria Intensiva) e Hospitalhaços, que atuam ludicamente em hospitais do interior de São Paulo há pelo menos três anos; de ambos os sexos e idade entre $21 \mathrm{e}$ 30 anos e médicos $(n=2)$ da área pediátrica hospitalar do Hospital São Francisco e que desempenham suas atividades no mesmo municipio citado.

Todos assinaram o Termo de Consentimento Livre e Esclarecido (TCLE) indicando a livre concordância e compreensão dos procedimentos a serem utilizados na pesquisa, sendo este um critério de inclusão. Este trabalho foi iniciado após aprovação do Comitê de Ética em Pesquisa da Faculdade Municipal Professor Franco Montoro tendo recebido parecer favorável sob o registro $\mathrm{n}^{\circ} 1.180 .848 \mathrm{e}$ seguiu todas as normas que regulamentam a pesquisa em seres humanos, contidas na Resolução 196/96 do Conselho Nacional de Saúde.

\section{o Instrumentos}

Para a coleta de dados, foi empregado um questionário semiestruturado com cinco perguntas abertas elaboradas pelos autores e aplicado a todos os contribuintes da pesquisa que englobassem a visão de suas atuações, assim como influências que percebem na criança hospitalizada e como as interações dos palhaços intervêm no tratamento, na recuperação e na internação da mesma. Junto ás respostas dos questionários, os palhaços e médicos relataram vivências compartilhadas às crianças internadas.

\section{o Procedimentos}

Para avaliar a eficácia da atuação lúdica do palhaço, esta pesquisa teve como base o método aplicado, sendo indutiva qualitativa. As análises dos dados foram, realizadas de forma qualitativa seguindo a metodologia da análise de conteúdo.

A aplicação do questionário ocorreu em horários agendados, em uma sala reservada do Hospital São Francisco que reunia condições para assegurar a privacidade dos participantes e a não interrupção do processo de coleta de dados.

Junto a cada participante foram esclarecidos os objetivos e o caráter voluntário e confidencial do estudo. $\mathrm{O}$ tempo de resposta de cada participante durou em média, 15 minutos e após a aplicação do questionário, foi realizada a análise de conteúdos, pelos investigadores envolvidos, sendo tabulados os resultados e comparados para averiguação e discussão dos mesmos. Nesta etapa foi levado em consideração a análise, categorização, descrição e interpretação das respostas como etapas essenciais desta metodologia, analisando a frequência de ocorrência de determinados termos, construções e referências no texto extraído das respostas ao questionário.

\section{RESULTADOS E DISCUSSÃO}

Constatou-se na maioria dos relatos obtidos que, a atuação lúdica do palhaço ocasiona uma alteração positiva no ambiente e no comportamento de crianças hospitalizadas e que possibilita a continuidade de uma infância alegre; é o que conota trechos dos relatos dos palhaços atuantes e médicos participantes da pesquisa, tais como: 1. "O palhaço no ambiente hospitalar é importante para que a criança continue a vivenciar atividades lúdicas que fazem parte de seu cotidiano, pois, é através da brincadeira que a criança expressa seus temores e desejos". 2. "O lúdico é capaz de resolver de forma inusitada determinadas situações, alterando a percepção do que se passa no hospital. O palhaço chama a atenção para aquilo que está saudável na criança doente, provocando alterações em seu estado de humor". 3. "O paciente pediátrico sofre uma grande mudança em seu cotidiano, o que faz com que a influencia do lúdico seja positiva, modificando o ambiente hostil, afastando a insegurança, o medo e os estranhamentos, o que gera no paciente uma sensação de bem estar. Tal mudança proporciona sensação de segurança, acolhimento e melhor adesão ao tratamento".

Os dados observados nesta pesquisa corroboram os resultados obtidos por Kumato et al. ${ }^{10}$ de que a ludicidade proporciona á criança uma relação afetiva com o mundo, sendo essencial para sua saúde, aumentando seu humor e permitindo uma melhor expressão de seus sentimentos.

Quando o lúdico FOI realizado tanto na presença do paciente quanto do acompanhante observou-se uma maior facilidade na condução dos procedimentos clínicos realizados pela equipe médica e pela equipe multidisciplinar do hospital, inclusive na aceitação do paciente, havendo uma mudança de percepção destes fatores conforme relatos: 1. "Nota-se a facilitação do trabalho desempenhado no cuidado com os pacientes, juntamente com a diminuição do estresse da rotina hospitalar". 2. "Os enfermeiros se tornam receptivos, os pacientes passam a aderir o tratamento com maior facilidade devido estarem mais alegres $e$ o ambiente de forma geral se torna mais leve e menos tenso". 3. "Percebe-se melhora do humor, da aceitação e da internação. Estas mudanças ocorrem tanto com o paciente quanto com a equipe". 4. "Tais atividades geram sentimento de alegria, descontração e bem estar, e ainda, contribuem para socialização entre os próprios pacientes e também entre os pacientes e a equipe hospitalar". 
Araújo e Guimarães ${ }^{12}$ retratam em sua pesquisa que a intervenção da figura do palhaço é capaz de facilitar o enfrentamento durante a doença, pois leva a criança a uma melhor alimentação e aceitação da mesma.

Porém, caso o palhaço atuante não esteja preparado e não tenha uma boa percepção da receptividade da criança, esta atuação pode ter consequências negativas segundo dados coletados: 1. "A brincadeira passa a ser algo sério, é preciso cuidado para perceber qual é a situação de cada paciente". 2. "O lúdico é incomum aos olhos de algumas crianças no ambiente hospitalar. O brincar e o jogar não tem hora para acontecer, depende dos olhares e da permissão da criança, se esta se recusa é importante que o palhaço recue, mas antes disso, ele tenta sutilmente uma aproximação". 3. "Há casos que a criança fica com medo da figura do palhaço, quando isso ocorre é preciso que se retire o nariz, a fim de facilitar o processo de aproximação. Também já ocorreram casos da criança se assustar pelo branco do jaleco, afinal, pessoas de branco vem a todo momento "furá-las", isso as assusta, assim, é preciso entender o momento da criança, ser maleável a sua aceitação, não acordá-la se estiver dormindo e se estiver com dor, procurar ser breve". 4. "Cada pessoa responde de maneira individual a situações diversas, o que justifica que alguns pacientes possam reagir de maneira negativa".

Algumas alterações de humor são percebidas tanto no paciente quanto no acompanhante, de forma nítida; fato este promove uma melhor compreensão da internação e dos procedimentos hospitalares. Determinadas experiências são descritas a seguir: 1. "Certa vez, uma paciente com câncer e depressão não falava a uma semana, pois só chorava. Quando recebeu a visita dos palhaços a paciente sorriu e falou. Vendo esta reação a filha que a acompanhava se emocionou devido á nítida mudança no comportamento da mãe". 2. "Em uma de nossas visitas, entramos no quarto de uma menina que estava jantando. O pai tentava alimenta-la, porém, a mesma se recusava a comer, devido estar com anemia profunda, fazia-se essencial a ingestão de legumes. Naquele dia o que ela tinha para comer era beterraba, foi ai que começamos a brincar com a criança, dizendo que a beterraba deixaria sua língua roxa e pedimos para ela fazer o teste. Ao comer, pegamos um espelho e mostramos como sua língua estava, ela sorriu, achou divertido, e quis comer mais, o que facilitou o processo e deixou o pai com um imenso sorriso". 3. "A criança chorava de forma desesperada, não aceitando a medicação e ficando brava com toda a equipe, o palhaço a distraiu e facilitou a administração medicamentosa".

Dessa forma, o lúdico é capaz de gerar a humanização hospitalar, favorecendo um ambiente mais alegre e de fácil interação, o que proporciona um melhor trabalho da equipe médica, pois, os palhaços relatam que o ambiente hospitalar e os profissionais se tornam receptivos, o que possibilita ao paciente ser visto por completo, levando em consideração seus sentimentos. Este passa a ser olhado mais do que como um doente, sendo visto como uma pessoa que precisa de cuidados e merece atenção. Tais aspectos foram ressaltados por um dos médicos entrevistado relatando a importância dessa mudança de rotina e do ambiente em que a criança se encontra e o quão importante o lúdico se torna para quebrar tal hostilidade, sendo este fator retratado por Jeammer ${ }^{16} \mathrm{e}$ Parcianello e Felin ${ }^{2}$.

Frente aos relatos médicos, é possível verificar que ambos reconhecem que a atuação no âmbito hospitalar repercute de forma positiva e facilitadora; proporcionando melhorias e maior aceitação no quadro doentio da criança.
Tal aspecto pode ser confirmado quando um dos médicos participantes da pesquisa relata que vivenciou uma situação em que uma criança comportava-se de forma resistente á ação medicamentosa, e no momento de atuação do palhaço humanizador, houve certa distração, levando - a ao riso, favorecendo á liberação de endorfina, permitindo uma melhor intervenção médica, sendo esses resultados relatados por Araújo e Guimarães ${ }^{12}$.

Diante de tais aspectos, um dos médicos entrevistados ressalta que os benefícios do lúdico proporcionam ainda, assimilação e aceitação da patologia gerando melhor adesão ao tratamento, o que consequentemente aumenta a autoestima e eleva os níveis de linfócitos NK, contribuindo para a melhora na imunidade.

Com o lúdico o tratamento se torna menos desagradável e o paciente passa a aceita-lo com maior facilidade, pois o palhaço é capaz de pegar algo que para a criança representa dor, como tomar uma injeção, e transformar este momento, levando-a á sorrir após a intervenção. De acordo com Motta e Enumo ${ }^{18}$, qualquer atividade recreativa pode ser considerada terapêutica quando auxilia na promoção do bem estar da criança, mesmo sendo uma atividade livre.

De modo geral verifica-se a necessidade da preparação do palhaço atuante, respeitando e compreendendo o momento da criança, algo que nos leva a um tema bastante extenso e que pode ser pesquisado posteriormente. Contudo, percebe-se que mediante ás adversidades que surgem fisicamente e afetivamente no ambiente hospitalar, é fundamental que haja incentivos e recursos ao trabalho do palhaço humanizador ${ }^{12}$. Ao buscar a opinião de médicos, tendo em vista sua influência e importância no contexto hospitalar, observou-se que a visão destes quanto ao lúdico vem de encontro ao posicionamento dos palhaços, ressaltando também a importância e benefícios do sorrir. Em contrapartida fatores limitantes foram encontrados, devido à indisponibilidade e a não adesão à participação da pesquisa por parte de alguns médicos, o que impossibilitou maior abrangência da pesquisa.

Pesquisas posteriores se tornam relevantes, podendo ampliar a amostra de médicos e palhaços participantes, buscando ainda a opinião do paciente, que representa papel fundamental neste processo, expandindo ainda mais o campo de pesquisa e conhecimento envolvendo o lúdico hospitalar.

\section{CONCLUSÃO}

A figura do palhaço mostrou o quão significativo podem ser suas ações, retirando a criança hospitalizada da atmosfera invasiva e dolorosa a qual foi submetida, levandoa á uma ressignificação do momento pelo qual esta passando. Este estudo mostrou que a atuação desses atores no ambiente hospitalar são potenciadoras do bem-estar e dos processos de recuperação do paciente pediátrico com a redução de processos álgicos, ansiedade, tristeza, resistência aos tratamentos e uma maior satisfação e melhor "qualidade de vida" no contexto hospitalar.

Muito embora os dados divulgados neste artigo se centrem num conjunto específico, eles direcionam para um olhar positivo por parte dos médicos entrevistados em referencia ao contributo desses palhaços na vivência da hospitalização infantil.

Desta forma, mais do que tratar a saúde exterior do paciente e proporcionar melhora de seus sintomas, o palhaço trata o interior, trazendo a tona sentimentos e anseios, reativando sonhos e esperança interrompidos pela internação, possibilitando uma transformação dos corredores 
hospitalares em um "palco", onde o espetáculo principal é a arte de fazer sorrir.

\section{REFERÊNCIAS}

1. Carvalho AM, Begnis JG. Brincar em unidades de atendimento pediátrico: aplicações e perspectivas. Psicologia em Estudo. 2006;11(1):109-17.

2. Parcianello AT, Felin RB. E agora doutor, onde vou brincar? Considerações sobre a hospitalização infantil. Barbarói. 2008; 28(1):147-66.

3. Vitorino SC, Linhares MBM, Minardi MRFL. Interações entre crianças hospitalizadas e uma psicóloga, durante atendimento psicopedagógico em enfermaria de pediatria. Estud Psicol. 2005; 10(2):267-77.

4. Bersch AAS. O brincar como fator potencializador da saúde ambiental no microssistema pediatria: uma análise bioecológica [dissertação]. Rio Grande: Universidade Federal do Rio Grande; 2005.

5. Oliveira SSG, Dias MGBB, Roazzi A. O lúdico e suas implicações nas estratégias de regulação das emoções em crianças hospitalizadas. Psicol Reflex Crit. 2003; 16(1):1-13.

6. Garbino A, Assis CR, Carmo DS, Meister D, Silva ECS, Acorci P. Brincar é coisa séria. Disponível em: https://psicologado.com/atuacao/psicologia-hospitalar/ brincar-e-coisa-seria. Acesso em: 04 de outubro 2016.

7. Machado MMP, Gioia-Martins D. A criança hospitalizada: espaço potencial e o palhaço. Bol Iniciação Científica Psicol. 2002, 3(1): 34-52.

8. Straub R. Nutrição, obesidade e transtorno da alimentação. In: Psicologia da saúde. Porto Alegre: Artmed, 2005. cap.7.

9. CONANDA. Resoluções do Conselho Nacional dos Direitos da Criança e do Adolescente. Disponível em: $<$ http://dh.sdh.gov.br/download/resolucoes-conanda/res1-a-99.pdf> Acesso em: 12 outubro 2016.

10. Kumamoto LHMCC, Gadelha ECM, Monteiiro FR, Silva LRMS, Leite MC, Santos RGC. Apoio à criança hospitalizada: uma proposta de intervenção lúdica. Rev Extensão Cidadã. 2006;1.

11. Valle ER, Ramalho MAN. O câncer na criança: a difícil trajetória. In Carvalho VA. (Org.). Temas em Psicooncologia. São Paulo: Summus; 2008.

12. Araújo TCCF, Guimarães TB. Interações entre voluntários e usuários em onco-hematologia pediátrica: um estudo sobre os "palhaços-doutores". Est Pesqui Psicol. 2009;9(3):632-47.

13. Ventura AF, Bobillo FS, Gil MD. A brincadeira que virou solidariedade - Os doutores da Alegria. Disponível em: $\quad$ http://www.unimep.br/ phpg/ mostraacademica/anais/4mostra/pdfs/289.pdf. Acesso em: 11 de outubro 2016.

14. Soares ALM. Palhaço de hospital: proposta metodológica de formação. [tese].Rio de Janeiro: PPGT-Unirio; 2007.

15. Warren B, Chodzinski R. An interview with Dr. Bernie Warren, clown doctor and founder of fools for health. Teaching and Learning. 2005; 2(3):7-10.

16. Jeammet P. Reynaud M. Consoli S. Manual de psicologia médica. Rio de Janeiro: Medsi; 2000.

17. Carmo MRC. O brincar no hospital: possibilidade de recuperação da saúde da criança e do adolescente. Disponível em: http://editorarealize.com.br/ revistas/fiped/trabalhos/Trabalho_Comunicacao_oral_idi nscrito_1258_3bc07d0d116e6e025a6470a4ff9a973e.pd. Acesso em 20 de agosto 2015.
18. Motta AB, Enumo SRF. Brincar no hospital: estratégia de enfrentamento da hospitalização infantil. Psicologia em Estudo. 2004; 9(1):19-28.

\section{CONFLITO DE INTERESSES}

Os autores declaram não haver conflitos de interesse.

\section{AUTOR PARA CORRESPONDÊNCIA}

\section{Anderson Martelli}

martellibio@hotmail.com

Submetido em 14/10/2016 Aceito em 05/12/2016 